\title{
Multiscale thermo-mechanical analysis of multi-layered coatings in solar thermal applications
}

\author{
F. Montero-Chacón ${ }^{1,}$, S. Zaghi ${ }^{2}$, R. Rossi², E. García-Pérez ${ }^{3}$, I. Heras-Pérez ${ }^{3}$, X. \\ Martínez ${ }^{2}$, S. Oller ${ }^{2}$ and M. Doblaré ${ }^{4}$. \\ ${ }^{1}$ Universidad Loyola Andalucía, Dpto. Ingeniería, Calle Energía Solar, 1, 41014, Seville, Spain. \\ ${ }^{2}$ Centre Internacional de Mètodes Numèrics a l'Enginyeria, C/ Gran Capità, s/n, 08034, \\ Barcelona, Spain \\ ${ }^{3}$ Abengoa Research, Calle Energía Solar, 1, 41014, Seville, Spain. \\ ${ }^{4}$ Group of Structural Mechanics and Materials Modelling (GEMM), Aragon Institute of \\ Engineering Research (I3A), University of Zaragoza, Spain
}

\begin{abstract}
Solar selective coatings can be multi-layered materials that optimize the solar absorption while reducing thermal radiation losses, granting the material long-term stability. These layers are deposited on structural materials (e.g., stainless steel, Inconel) in order to enhance the optical and thermal properties of the heat transfer system. However, interesting questions regarding their mechanical stability arise when operating at high temperatures. In this work, a full thermomechanical multiscale methodology is presented, covering the nano-, micro-, and macroscopic scales. In such methodology, fundamental material properties are determined by means of molecular dynamics simulations that are consequently implemented at the microstructural level by means of finite element analyses. On the other hand, the macroscale problem is solved while taking into account the effect of the microstructure via thermo-mechanical homogenization on a representative volume element (RVE). The methodology presented herein has been successfully implemented in a reference problem in concentrating solar power plants, namely the characterization of a carbon-based nanocomposite and the obtained results are in agreement with the expected theoretical values, demonstrating that it is now possible to apply successfully the concepts behind Integrated Computational Materials Engineering to design new coatings for complex realistic thermo-mechanical applications.
\end{abstract}

Keywords: multiscale analysis $\cdot$ thermo-mechanical homogenization $\cdot$ finite element method • representative volume element $(\mathrm{RVE}) \cdot$ molecular dynamics $\cdot$ solar selective coatings 


\section{Introduction}

One of the most difficult challenges in Solar Thermal Electricity (STE) power plants is the increase in the operating temperature beyond current limits in order to boost the efficiency of the cycle in concentrated solar power (CSP) plants.

There are two major CSP plant configurations, namely central receiver systems (CRS) and parabolic trough (Figure 1a and b, respectively). A key component in both configurations is the receiver tube, through which solar heat radiation is transferred to the so-called heat transfer fluid (HTF), increasing its temperature before feeding the power block [1]. In the former configuration, tubes are arranged within an exposed cavity; while in the latter, the absorber tube is located at the focal point of the collector, usually inside the vacuum chamber conformed by another external glass tube. The thermal performance of this component strongly depends on the optical and thermo-mechanical properties of the tube materials.
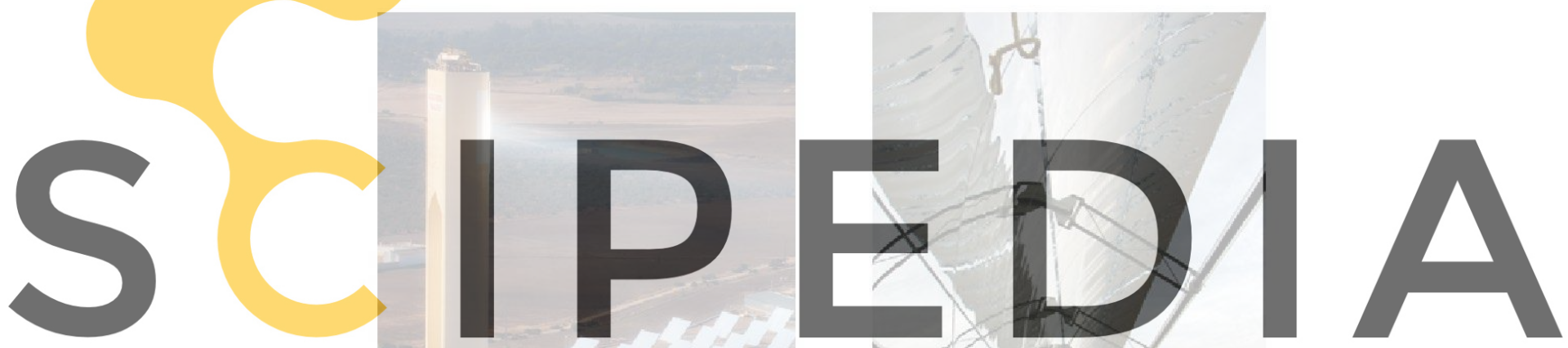

Register for free at https//www.scipedia.com to download the version without the watermark

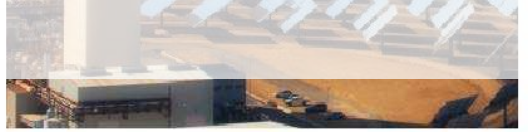

(a)

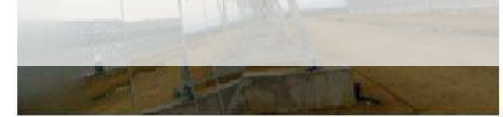

(b)

Figure 1. Solar selective coatings are present in two main concentrating solar power (CSP) plant configurations: a) central receiver systems and b) parabolic trough absorber tubes.

The final goal of the receiver tube is therefore to heat the HTF that will eventually run (directly or by means of steam via an intermediate heat exchanger) the turbine. Thus, the purpose of the tube is to absorb as much solar radiation as possible, conduct it, and finally heat the HTF by forced convection [2].

Solar selective coatings (SSCs) [3, 4] are multi-layered materials that optimize the solar absorption while reducing the thermal radiation losses, granting the long-term structural stability. A variety of thin film architectures can be exploited as SSCs for obtaining spectral selectivity. One of the most efficient configurations is based on a multi-layer stack, whose composing layers are usually arranged as depicted in Figure 2, and described in the following [5]: 
a) an antireflective (AR) layer (e.g., $\mathrm{Al}_{2} \mathrm{O}_{3}, \mathrm{SiO}_{2}$ ), transparent to solar radiation to increase solar absorption and chemically stable in air at high temperature so as to reduce corrosion;

b) an absorber layer (e.g., transition-metal nanocomposites, cermets), which must offer optimal optical properties regarding absorption and conductivity as well as thermomechanical stability;

c) an infrared reflective (IR) layer (e.g., metal nitrides) beneath the absorber, with high reflectivity in the infrared region to reduce emissivity; and, finally,

d) a substrate (e.g., stainless steel or Ni-based alloys) layer, that hosts the HTF and provides mechanical resistance.

The composing materials of each of these layers are typically chosen (or designed) according to their particular ontical properties (Figure 3); therefore, major efforts by materials scientists in Abengoa Research have been devoted in the last years to the optical enhancement of SSCs [5]. Howeyer, important questions regarding their thermo-mechanical stability have to be considered.
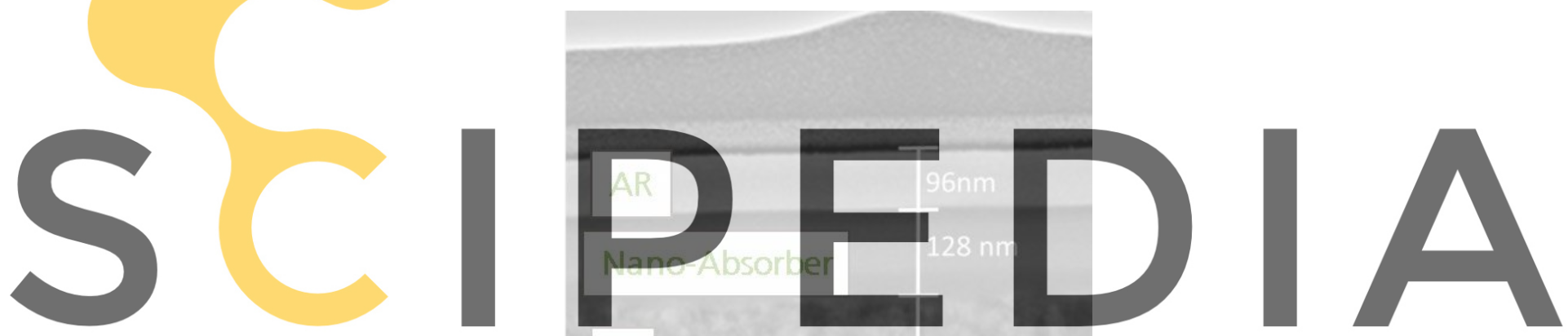

Register for free at https//www.scipedia.com to download the version without the watermark $100 \mathrm{~nm}$

Figure 2. Cross section scanning electron image of a complete selective stack structure developed for the present application. The stack consists (from substrate to the surface) of an infrared (IR) layer, an absorber nanocomposite coating (nanoabsorber), and an antireflective (AR) top film.

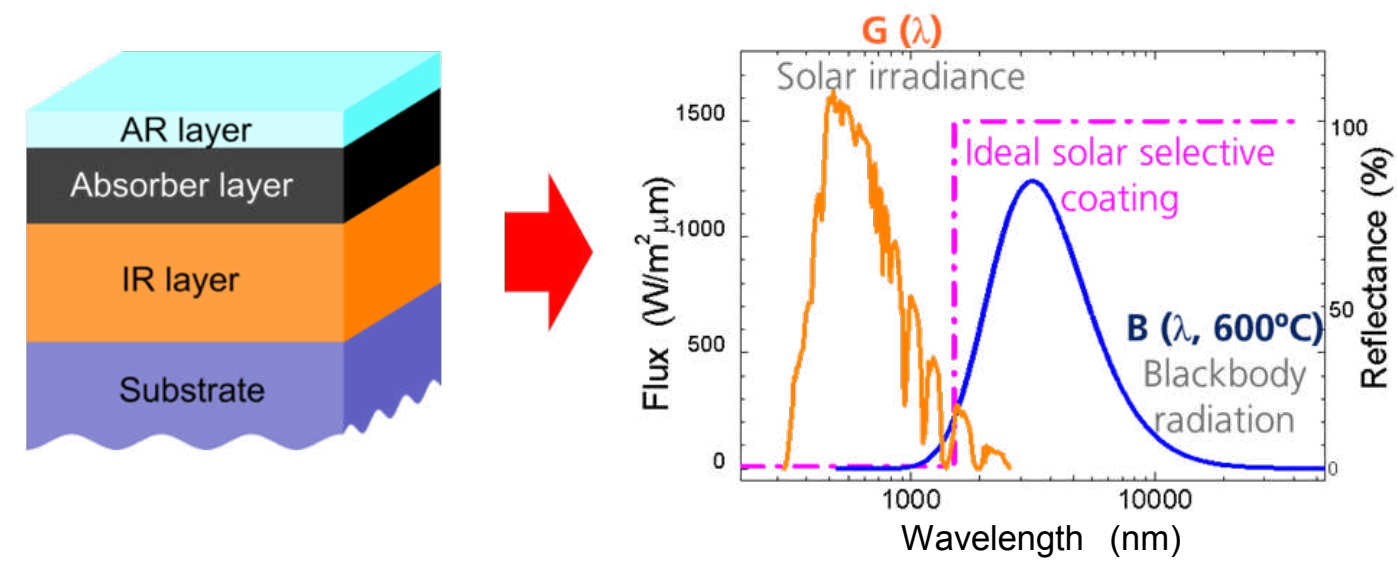

Figure 3. Optical properties of the solar selective coating developed for the present study. 
In this work, a bottom-up multiscale methodology (Figure 4), developed within the Abengoa's Virtual Materials Design (VMD) project [6], is reported in order to establish a virtual laboratory for testing new structured materials (e.g., SSCs) for renewable energy solutions. In this virtual laboratory, SSCs macroscopic properties required by the continuum models (usually modeled and solved by means of finite element analyses, FEA) are obtained from nanoscale molecular dynamics (MD) simulations in a succession of progressively higher scales with their corresponding interfaces. In this particular work, and in order to show the capabilities of this new tool, a new nanoabsorber layer composed by an amorphous carbon (a-C) matrix reinforced with titanium carbide (TiC) nanoparticles is analyzed. In this sense, original MD results are presented herein, especially for the case of a-C for which a large dispersion in the thermal and mechanical properties is found in the literature $[7,8,9]$.

Multiscale analysis has been presented as a powerful tool in order to analyze complex heterogeneous media such as composite materials $[10,11]$. Moreover, it has been applied in twolevel continuum thermo-mechanical problems $[12,13]$ or, more recently, covering even three continuum scales $[14,15]$. With regard to applications, multiscale thermo-mechanical schemes have been successfully used in RVE-based problems (i.e., micro to macro transition) like, for
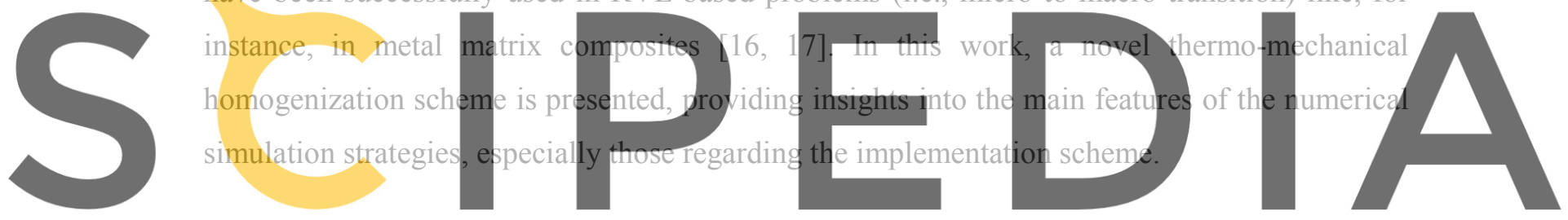

Bottom-up approach

Register for free at https//wwww.scipedia.com to download the version without the watermark

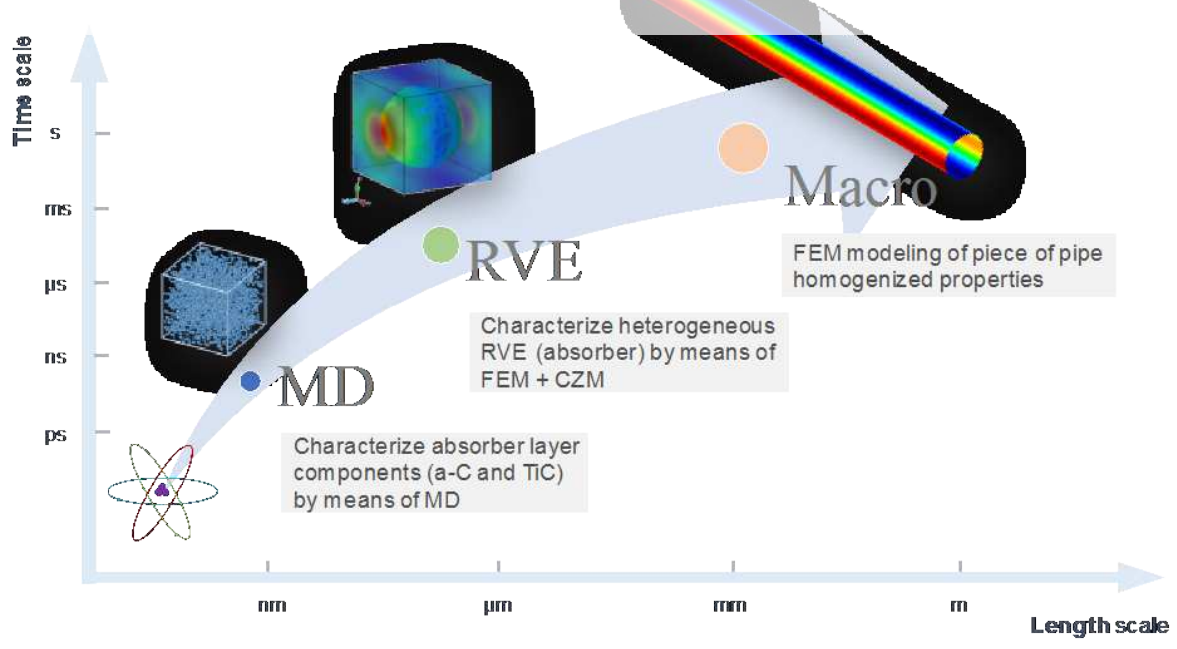

Figure 4. From atoms to structures: bottom-up multiscale strategy used in the thermo-mechanical analyses of receiver tubes. 
Finally, the thermo-mechanical behavior of the different layers in a piece of the receiver tube is analyzed at the macroscale, providing useful information for the design of this type of components while validating the presented multiscale methodology and demonstrating that it is possible to conduct multiscale analyses, from atoms to structures, in a three-scale scheme. However, it must be remarked that, in the presence of other conditions such as finite strains, strainrate dependence, or damage localization, other alternative schemes $[15,18]$ are more adequate.

\section{The Virtual Materials Design platform}

The Virtual Materials Design (VMD) project was firstly included in Abengoa Research's Strategic Research Agenda in 2012 [6, 19] with the main purpose of contributing to the development of a multipurpose simulation platform able to model and simulate the behavior of complex materials, obtaining the material properties from the most fundamental ones. The longterm goal of the project was to cover a wide range of scales, from the atomistic to the macroscopic scales in fields like structural mechanics, thermal problems, optoelectronics, and electrical applications, with the final aim of helping in the design of new materials. To summarize, the specific objectives of the VMD project were:
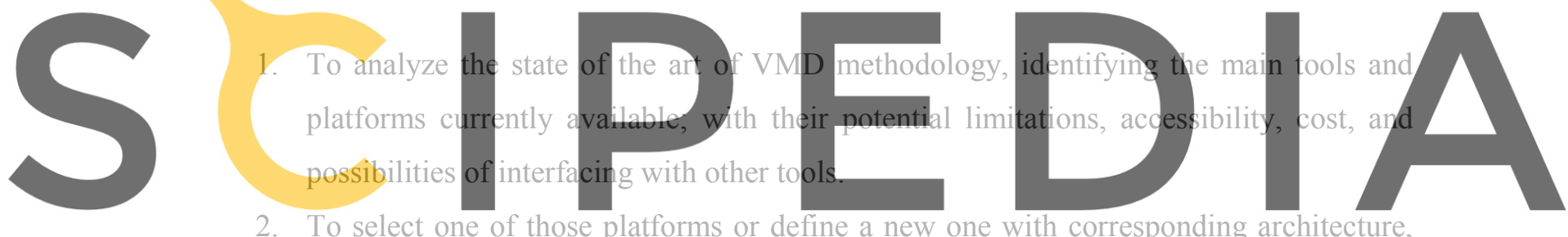

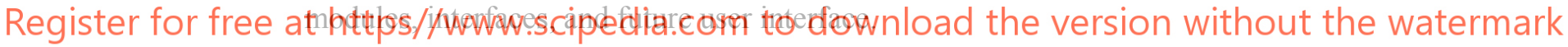

3. To develop an initial module for interfacing with molecular dynamics software available, able to analyze the thermo-mechanical properties of a new material with respect to others under different conditions.

4. To develop an initial module for interfacing with finite element continuum mechanics software available, able to analyze the effect on the macroscopic mechanical properties of the volume ratio of the components and their distribution into the microstructure.

5. Application of these two modules to some reference problems for assessing their capabilities.

As mentioned above, the main goal of this project was to provide a computational framework in order to create and study new innovative materials with targeted properties, optimizing the design process. This enlightening approach would allow the design of special-purpose materials, tailoring their macroscopic properties from their particular composition and microstructure while, at the same time, reducing the number of characterization tests and expensive prototypes. Likewise, along this complex process, completely novel materials may be found. 
So far, great efforts have been devoted to numerical methods for the analyses of the different scales: nano, micro, meso, and macro [20, 12, 21, 22, 14]. However, coupling these scales still faces many difficulties, especially regarding the optimal procedure to share material properties between scales and in terms of the computational cost of performing a fully coupled multiscale analysis. In this sense, the challenge ahead is not only to couple a few scales, but to couple all of them to solve real engineering problems. It is within this context that the VMD project followed an Integrated Computational Materials Engineering (ICME) approach [20, 23], which aim was to pass from the traditional "handbook approach" philosophy to a holistic one in materials design, relying on current computational capabilities.

\section{Molecular Dynamics characterization of absorber layers}

The results at the lowest scale obtained in this work are achieved using molecular dynamics (MD) simulations, using the GiD-Lammps code, which is a graphic user interface (GUI) connected to Lammps [24] and developed by CIMNE within the VMD project [6, 25].

The accuracy of atomistic MD simulations directly depends on the use of appropriate
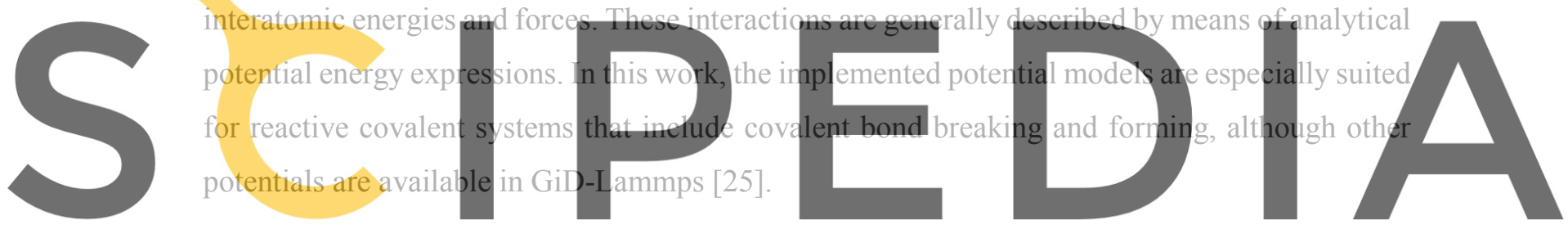

3.1. Interaction potentials for transition metal nanocomposites

Register for free at https//WwW.scipedia.com to download the version without the watermark The first interaction potential used in this work is the so-called reactive empirical bond-order,

REBO, which is able to model chemical reactions especially those including carbon structures [26]. This potential has reactive capabilities and only describes short-ranged C-C interactions $\left(r_{i j}<2 \AA\right)$. These interactions have strong coordination-dependence through a bond order parameter, which adjusts the attraction between the $i$ and $j$ atoms, based on the position of other nearby atoms.

Unfortunately, the REBO potential is not able to allow for long-range atomic interactions, which are of great interest in carbon systems. For this reason, a next generation REBO potential that overcomes this problem is used, the so-called adaptative intermolecular reactive empirical bond order, usually referred to as AIREBO [27]. This potential includes a series of switching functions representing the short-ranged Lennard-Jones repulsion and torsional potentials that are particularly useful for modeling amorphous carbon.

The third interaction potential that is used in this work is the $2^{\text {nd }}$ generation modified embedded atom method, 2NN-MEAM $[28,29]$, by modifying the original MEAM to partially consider $2^{\text {nd }}$ 
nearest-neighbor atom interactions and to remove some critical shortcomings in the former potential. It is thus suitable for modeling metal and alloys with fcc, bcc, hcp, and diamond cubic structures, as well as covalently bonded materials like silicon and carbon. The parameters used in this work are taken from [30, 31].

\subsection{Mechanical properties characterization}

The interaction potentials defined above are used in order to characterize the system of study. Regarding the mechanical properties, we will focus on elastic constants, stress-strain curves, and the coefficient of thermal expansion (CTE). The procedure to analyze these properties is described next.

The elastic constants characterize the stiffness of a material. The formal definition is provided by the linear relation that holds between the stress and strain tensors in the limit of an infinitesimal deformation, thus, in tensorial notation:

$$
\sigma_{i j}=C_{i j k l} \varepsilon_{k l}
$$

with $\sigma_{i j}$ and $\varepsilon_{k l}$ the second-order stress and strain tensors, respectively, and $C_{i j k l}$ the fourth-order
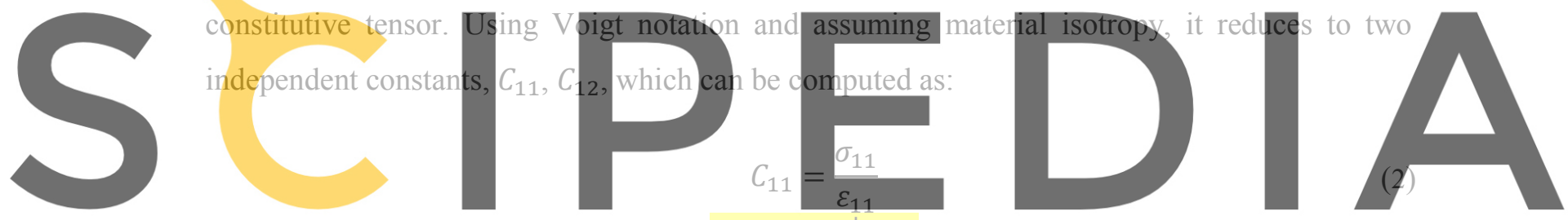

Register for free at https//www.scipedia.com to download the version without the watermark by means of a uniaxial strain test, loaded in the $x$ direction and prescribing $\varepsilon_{22}=\varepsilon_{33}=0$.

In turn, under the same assumptions, such constants can be used to compute the elastic modulus, $E$, and the Poisson's ratio, $v$, as:

$$
\begin{gathered}
E=\frac{\left(C_{11}+2 C_{12}\right)\left(C_{11}-C_{12}\right)}{\left(C_{11}+C_{12}\right)} \\
v=\frac{C_{12}}{C_{11}+C_{12}}
\end{gathered}
$$

The stress-strain curve describes the relationship between the stress and strain that a particular material displays. It is unique for each material and is found by recording the amount of deformation (strain) at several intervals of tensile or compressive loading (stress) or vice versa. 
On the other hand, thermal expansion is the tendency of matter to change in volume in response to a change in temperature, and it is taken into account by the coefficient of thermal expansion (CTE), defined as the fractional change in length per degree of temperature change:

$$
\alpha=\frac{1}{L} \cdot \frac{d L}{d T}
$$

where $L$ is a reference length .

\subsection{Thermal properties}

With regard to thermal properties, we focus on the specific heat and the thermal conductivity, which can be computed by MD using the interaction potentials specified in section 3.1.

The specific heat, $C_{p}$, is the amount of heat needed to raise the temperature $1 \mathrm{~K}$ per kilogram of substance. This is computed at constant pressure for a specific temperature range:

$$
d H=C_{p} d T
$$

On the other hand, the thermal conductivity, $k$, is a measure of the tendency of a material to
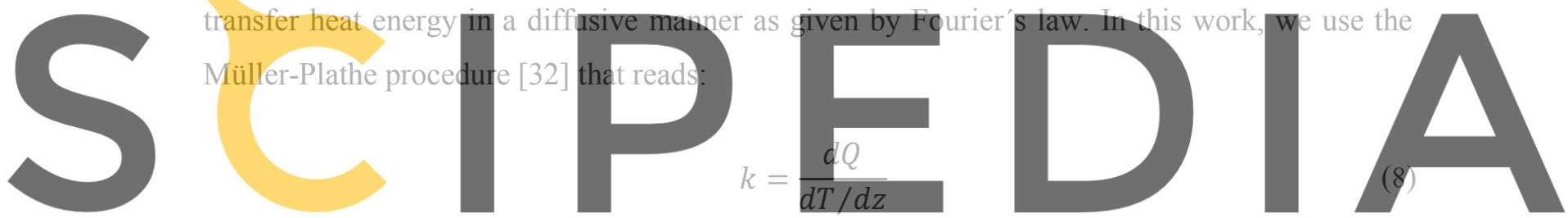

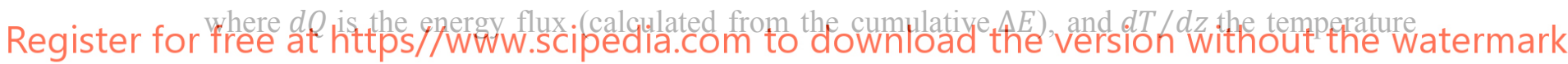
gradient.

\section{Thermo-mechanical multiscale method}

First order thermo-mechanical homogenization consists in obtaining the material response of the macroscale structure from the analysis of a representative volume element (RVE) that provides the material thermo-mechanical performance at the microscale level. This section summarizes the main concepts and basic equations of a general multiscale method, while the following section proposes a simplified procedure to solve engineering structures with an affordable computational cost.

In a multiscale procedure, the strain and thermal gradient obtained when analyzing the macroscopic structure are used to define the boundary conditions for the microscale problem. The solution of the problem at the microscale, under such conditions, acts as an equivalent constitutive law for the macroscale, as it provides material parameters: stiffness and conductivity, and the response to the conditions applied: stresses and temperature. This equivalent constitutive law is 
used in all the Gauss points of the macroscopic model in order to obtain the global response of the structure. In case of having a non-linear performance of the microscopic model, a general approach will lead to an iterative procedure in which the RVE must be solved for different boundary conditions until both scales reach equilibrium, ensuring consistency between the microand macroscale solutions [33].

The first-order homogenization technique developed assumes a scale separation from the macro- to the microscale [34], that is, the characteristic length of the microscale $l$ should be much smaller than the length of the macroscale elements, $L: l<<L$. Also, for the sake of simplicity, the procedure assumes small displacements and a quasi-static structural performance in both scales, macro and micro.

Therefore, a general multiscale algorithm can be defined by three main stages. The first step is the macro to micro transition, where the macroscopic strain and temperature gradient at any point of the macroscopic mesh are transferred to the microscale. Afterwards, the boundary value problem (BVP) is solved at the microscale to obtain its mechanical and thermal response. Finally, the macroscale parameters are obtained through the micro-to-macro interfacing step (Figure 5).
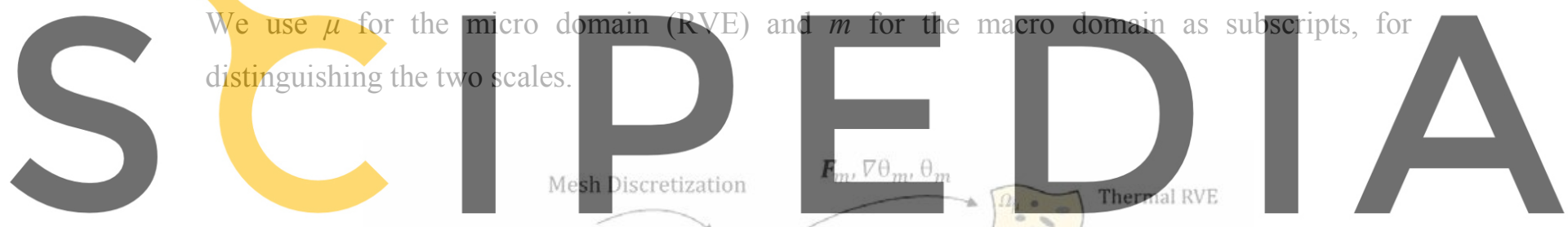

Register for free at https//www.scipedia.com to download the version without the watermark

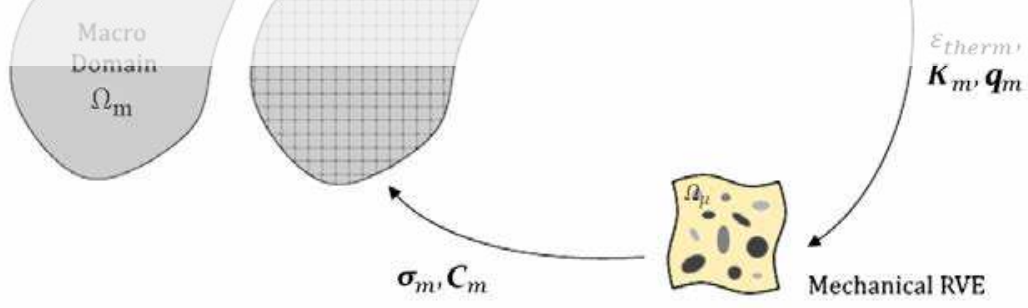

Figure 5. Thermo-mechanical multiscale method.

\subsection{Macro to micro transition}

At the macro level, the starting point for a kinematically based computational homogenization method is the assumption that the mechanical strain tensor, $\boldsymbol{\varepsilon}_{m}$, at each point of the macroscale domain, $\Omega_{m}$ (where the position is defined through the vector $\boldsymbol{x}_{m}$ ), and at a certain instant $t$ can be obtained as the volume average of the microscopic mechanical strain field, $\boldsymbol{\varepsilon}_{\mu}$, defined at each point of the microscale domain, $\Omega_{\mu}$ (where the position is defined through the vector $\boldsymbol{x}_{\mu}$ ), and at the same time $t$ as: 


$$
\boldsymbol{\varepsilon}_{m}\left(\boldsymbol{x}_{m}, t\right)=\frac{1}{V_{\mu}} \int_{\Omega_{\mu}} \boldsymbol{\varepsilon}_{\mu}\left(\boldsymbol{x}_{\mu}, t\right) d V
$$

The microscopic strain field can be expressed as the symmetric gradient of the microscopic displacement field, $\boldsymbol{u}_{\mu}=\left(u_{\mu}^{x}, u_{\mu}^{y}\right)$ :

$$
\boldsymbol{\varepsilon}_{m}\left(\boldsymbol{x}_{m}, t\right)=\frac{1}{V_{\mu}} \int_{\Omega_{\mu}} \nabla^{s} \boldsymbol{u}_{\mu} d V
$$

In the same way we can define the macro temperature gradient, $\nabla \theta_{m}$, at each point $\boldsymbol{x}_{m}$ and at each time $t$, as the volume average of the microscopic temperature gradient, $\nabla \theta_{\mu}$, defined at each point $\boldsymbol{x}_{\mu}$ of the microscale domain and at each time $t$ :

$$
\nabla \theta_{m}\left(x_{m}, t\right)=\frac{1}{V_{\mu}} \int_{\Omega_{\mu}} \nabla \theta_{\mu}\left(x_{\mu}, t\right) d V
$$

Without loss of generality, we can decompose the microscale displacement and temperature as:
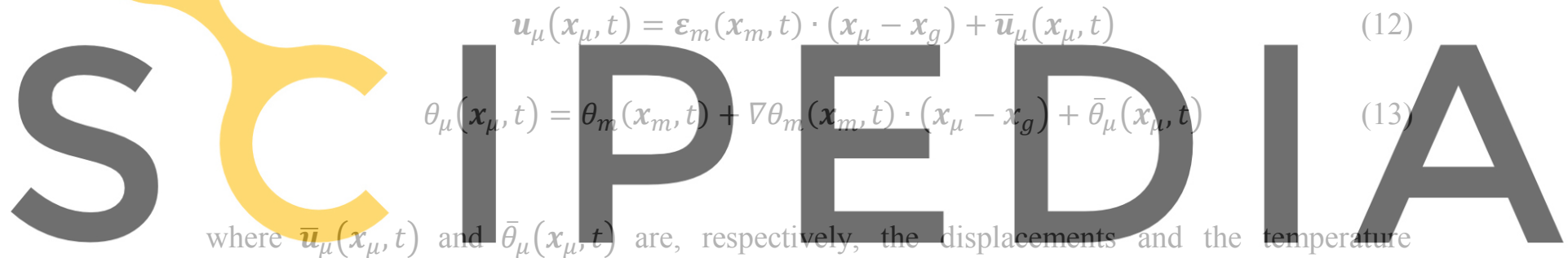

Register for free at hitups//WwWw.scipedia.com to download the version without the watermark t.

Finally, we have to define the corresponding boundary conditions on the microscale. For the displacement and temperature fluctuation fields, we use periodic boundary conditions since they generally provide an intermediate and more exact response compared to other type of boundary conditions, as is described in $[35,36,37,38]$ :

$$
\begin{gathered}
\overline{\boldsymbol{u}}_{\mu}\left(\boldsymbol{x}_{\mu}^{+}, t\right)=\overline{\boldsymbol{u}}_{\mu}\left(\boldsymbol{x}_{\mu}^{-}, t\right) \forall \operatorname{pair}\left\{\boldsymbol{x}_{\mu}^{+}, \boldsymbol{x}_{\mu}^{-}\right\} \in \partial \Omega_{\mu} \\
\bar{\theta}_{\mu}\left(\boldsymbol{x}_{\mu}^{+}, t\right)=\bar{\theta}_{\mu}\left(\boldsymbol{x}_{\mu}^{-}, t\right) \forall \operatorname{pair}\left\{\boldsymbol{x}_{\mu}^{+}, \boldsymbol{x}_{\mu}^{-}\right\} \in \partial \Omega_{\mu}
\end{gathered}
$$

being $\boldsymbol{x}_{\mu}^{+}$and $\boldsymbol{x}_{\mu}^{-}$two opposite points on the RVE boundary $\partial \Omega_{\mu}$.

\subsection{Solution of the thermal microscale BVP}

Using the Fourier equation for heat transfer, the equilibrium of the microscale is achieved when the following condition is verified 


$$
\nabla \theta_{m} \cdot \boldsymbol{q}_{m}=\frac{1}{V_{\mu}} \int_{\Omega_{\mu}} \nabla \theta_{\mu} \cdot \boldsymbol{q}_{\mu} d V
$$

taking $k_{i}$ as a component of conductivity matrix and $q_{i}$ as a component of heat flux vector. We can define $q_{i}$ as:

$$
q_{i}=-k_{i} \frac{\partial \theta}{\partial i} \text { with }(i=x, y, z)
$$

\subsection{Solution of the mechanical microscale BVP}

For the mechanical problem, the Hill-Mandel principle of macro-homogeneity establishes that the macroscopic stress power, product of macro stress tensor, $\sigma_{m}$, and the macro strain rate, $\bar{\varepsilon}_{m}$, must be equal to the microscopic stress power over the RVE averaged on the volume domain. Hence:

$$
\boldsymbol{\sigma}_{m}: \overline{\boldsymbol{\varepsilon}}_{m}=\frac{1}{V_{\mu}} \int_{\Omega_{\mu}} \boldsymbol{\sigma}_{\mu}: \overline{\boldsymbol{\varepsilon}}_{\mu} d V
$$

In order to obtain the strain field for the thermo-mechanical homogenization we need to add the contribution from the equilibrium of the thermal RVE:

$$
\boldsymbol{\varepsilon}_{\mu, \text { thermal }}=\alpha\left(\theta_{\mu}-\theta_{a m b}\right) \mathbf{I}
$$

where $\alpha$ is the coefficient of thermal expansion, $\boldsymbol{\varepsilon}_{\mu, \text { thermal }}$ is the thermal strain contribution, $\theta_{a m b}$ is the environment temperature, and $\mathbf{I}$ the identity tensor. Thus, the total microscale strain can be obtained adding the mechanical strain part, $\boldsymbol{\varepsilon}_{\mu, \text { mechanical }}$ :

$$
\boldsymbol{\varepsilon}_{\mu}=\boldsymbol{\varepsilon}_{\mu, \text { mechanical }}+\boldsymbol{\varepsilon}_{\mu, \text { thermal }}
$$

\subsection{Micro to macro transition}

Following the solution of the BVP for each case, we get the homogenized macroscopic stress tensor and the macroscopic heat flux vector. In addition, we can obtain the homogenized tangent operator and the macroscopic conductivity matrix.

The homogenized macroscopic stress tensor can be obtained as the microscopic stress field of the RVE averaged on the volume as:

$$
\boldsymbol{\sigma}_{m}=\frac{1}{V_{\mu}} \int_{\Omega_{\mu}} \boldsymbol{\sigma}_{\mu}\left(\boldsymbol{x}_{\mu}, t\right) d V
$$

Similarly, the homogenized macroscopic heat flux vector, $q_{m}$, can be evaluated in the same way through the microscopic heat flux vector of the RVE:

$$
\boldsymbol{q}_{m}=\frac{1}{V_{\mu}} \int_{\Omega_{\mu}} \boldsymbol{q}_{\mu}\left(\boldsymbol{x}_{\mu}, t\right) d V
$$




\subsection{Material homogenized properties}

The macroscopic constitutive relation defined by the homogenized properties of the RVE can be obtained after the solution of the microscale BVP.

Assuming the equilibrium of the microscale expressed as:

for the mechanical part; and

$$
\int_{\Omega_{\mu}} \boldsymbol{\sigma}_{\mu}: \nabla^{s} \overline{\boldsymbol{u}}_{\mu} d V=0
$$

$$
\int_{\Omega_{\mu}} \boldsymbol{q}_{\mu} \nabla \theta_{\mu}\left(\boldsymbol{x}_{\mu}, t\right) d V=0
$$

for the thermal one. As is described in [22] and [39], the homogenized constitutive tensor $\boldsymbol{C}^{H}$ can be defined as:

$$
\boldsymbol{C}^{H}=\frac{1}{V_{\mu}} \int_{\Omega_{\mu}} \boldsymbol{C}_{\mu} d V
$$

where $\boldsymbol{C}_{\mu}$ is the material constitutive tensor of the RVE.

The evaluation of the homogenized constitutive tensor is performed via a perturbation method. For each column $j$ of the constitutive tensor, a small strain perturbation $\left(\delta \overline{\boldsymbol{\varepsilon}}_{j}\right)$ is applied to the RVE in order to obtain, along with equation (23), a perturbed stress tensor $\left(\delta^{j} \overline{\boldsymbol{\sigma}}\right)$. The $j$ columns of the homogenized constitutive tensor can be obtained as:

$$
\boldsymbol{C}_{j}^{H} \equiv \frac{\delta^{j} \overline{\boldsymbol{\sigma}}}{\delta \overline{\boldsymbol{\varepsilon}}_{j}}
$$

Following an analogous procedure, the homogenized conductivity matrix can be defined as:

$$
K^{H}=\frac{1}{V_{\mu}} \int_{\Omega_{\mu}} K_{\mu} d V
$$

where $K_{\mu}$ is the material conductivity matrix of the RVE.

The microstructure BVP is solved imposing small temperature perturbations in each $j$ direction, using equations (19) and (24). This problem provides a perturbed flux vector $\left(\delta^{j} \overline{\boldsymbol{q}}\right)$, that can be used to calculate the $j$ column of the conductivity matrix:

$$
K_{j}^{H} \equiv \frac{\delta^{j} \bar{q}}{\delta \bar{\theta}_{j}}
$$

\section{Multiscale procedure}

This work aims to demonstrate that it is possible to conduct multiscale analyses of real structures, obtaining the material performance from the atomic scale. 
Despite the recent improvements on multiscale methods, most of them are based on obtaining material properties from molecular dynamics, or on coupling the macroscale (or structural) model with a mesoscale model that can account for the interaction among components in a composite $[10,11,40,41,42]$. Coupling these two scales already represents a significant computational cost as shown by Otero et al. in [43]. Besides, most of these analyses are conducted in the linear range because a non-linear analysis of a structure using a homogenized approach increases even more the computational cost, unless some strategy is used in order to reduce the number of time intervals in which the RVE has to be analyzed, such as the one presented in [39].

In the following, a simplified multiscale procedure to characterizing the thermo-mechanical response of a macro structure is proposed, moving from molecular dynamics to the macro-scale. The procedure provides all the advantages of multiscale analyses, in terms of material thermomechanical characterization, with an affordable computational cost. This scheme assumes that linear behavior is usually considered during the first stages of the design process for most engineering structures. In this case, the iterative procedure defined in section 4 is no longer needed, as the material properties will remain constant during the whole analysis. Therefore, it is possible to design the structure (or the material) by only using a bottom-up approach. Afterwards, the procedure offers the possibility of analyzing certain strategic RVEs, based on the results obtained from the macro-structure, in order to obtain their thermo-mechanical performance under the applied load regime (top-down analysis). This procedure is shown schematically in Figure 6 and is described in detail next:

1. The thermo-mechanical properties of the base materials used in the structural component are obtained from MD analysis, following the procedure shown in section 3.

2. The mesoscale of the structural material is analyzed using an RVE, in which the material properties of the different components are obtained from MD. The RVE is used to obtain the homogenized properties of the composite, as it has been shown in the previous section.

3. The macro structure is simulated with a finite element model that uses the material properties obtained from the RVE. These properties are not modified during the structural analysis. Therefore, the simulation is conducted assuming that the material performance of the RVE is linear, since the material thermo-mechanical properties are not modified even if the stresses exceed the failure threshold value.

4. Although the analysis conducted for the macro-structure does not take into account the nonlinear performance of the material, it is possible to study the material response once the macro-analysis has been completed. This is done by selecting the elements with larger stresses, or those placed in strategic locations, and applying the load history (e.g., strains and temperatures) obtained from the structural analysis onto the RVE. With these 
simulations, it is possible to check whether any of these materials has failed during the simulation and its corresponding failure mode.

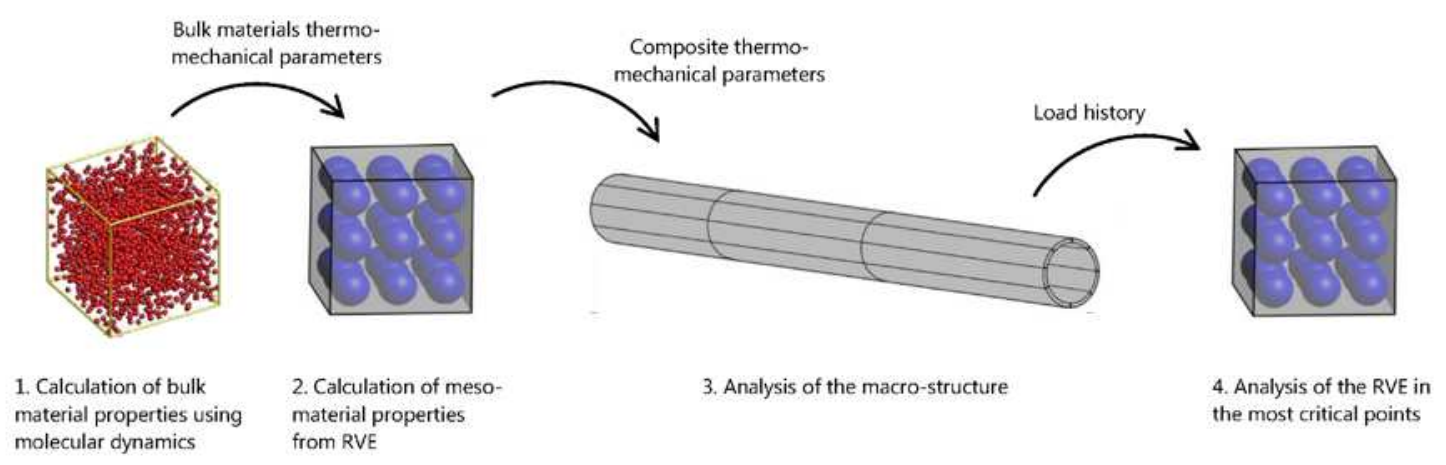

Figure 6. Multiscale thermo-mechanical procedure used in the current approach.

\section{Multiscale thermo-mechanical analysis of absorber tubes}

The present example was selected as a proof-of-concept test case for the proposed multiscale solution procedure. The objective is thus to test the capability of the proposed algorithm in capturing the physical behavior of interest.

Let us consider the case of an asymmetric temperature distribution in a receiver tube caused by the projection of the solar radiation on one half of the tube. This problem can be easily solved using the FEM, considering radiation and convection on the exposed half, and only convection on the other. This asymmetry in the temperature field will result in the deformation of the tube in bending. This temperature distribution can be applied to the different layers in the SSC, paying special attention to the absorber layer, which is critical. Figure 7 shows a scheme of this validation example.

The receiver tube substrate is made of Inconel, with the following layers: a) alumina as the AR layer; b) an amorphous carbon (a-C) matrix with embedded titanium carbide (TiC) particles as the nanoabsorber; and c) titanium nitride (TiN) as the IR layer. 


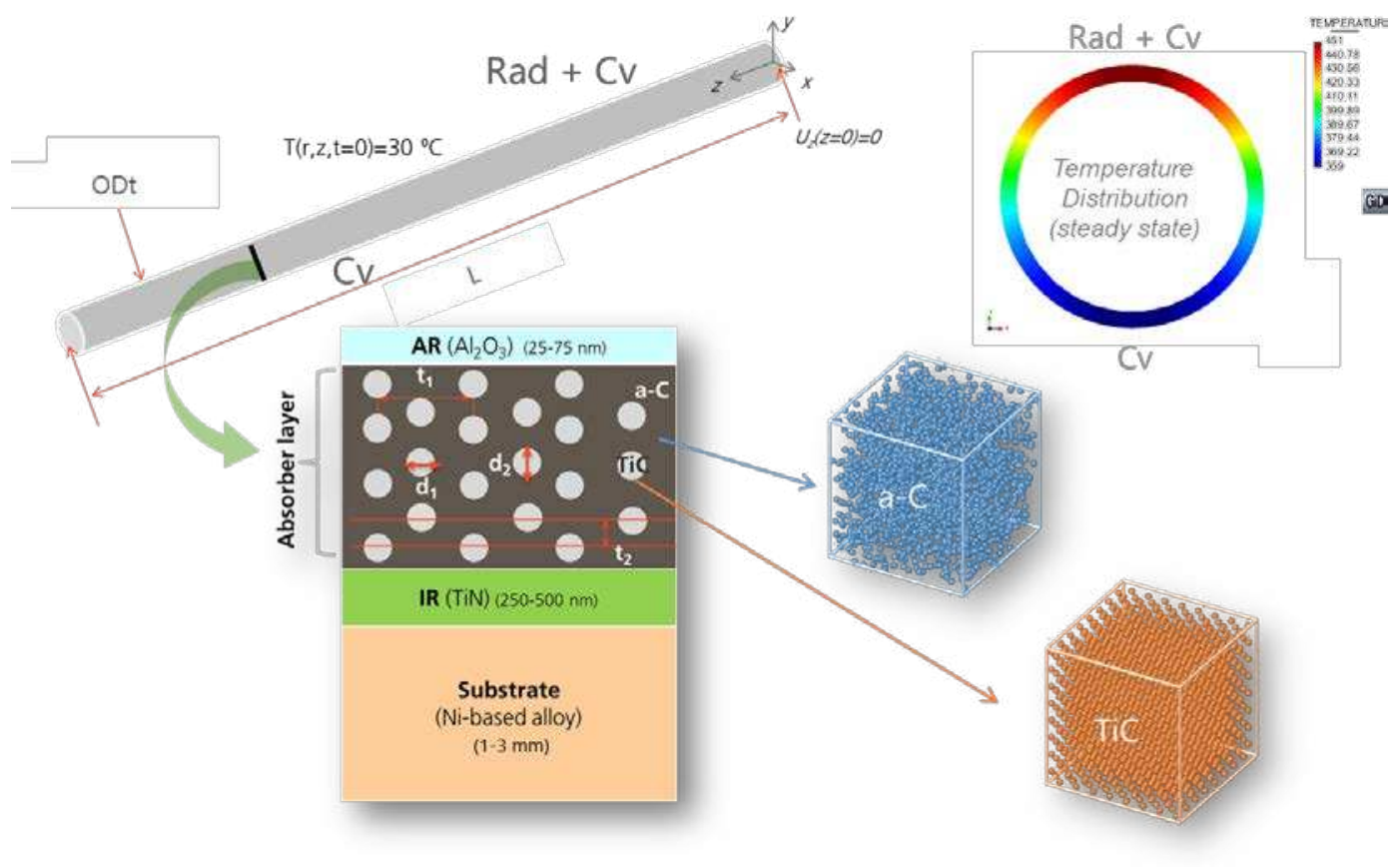

Figure 7. Proof-of-concept test case: multiscale analysis of absorber tubes with SSCs.

\subsection{MD characterization of a-C/TiC nanoabsorbers}

The simulated a-C systems were prepared at $2.6 \mathrm{~g} / \mathrm{cm}^{3}$ by quenching samples of molten carbon, as proposed by Stuart et al. [7], and using the three potentials described in section 3. The systems were heated to $5000 \mathrm{~K}$ with a Nosé-Hoover thermostat and equilibrated at that temperature for $100 \mathrm{ps}$. The liquid samples were then cooled down to $300 \mathrm{~K}$ over $100 \mathrm{ps}$. Following this, the systems were equilibrated for a further $50 \mathrm{ps}$ to anneal any transient structures (Figure 8). Finally, we calculated the temporal averages over another $50 \mathrm{ps}$.

Figure 9 shows the radial distribution function (RDF), which describes how density varies as a function of distance from a reference atom. Comparing Figure $9 \mathrm{a}$ and $\mathrm{b}$, it can be concluded that the agreement between the RDFs using the AIREBO and the 2NN-MEAM potentials with previous simulation data is quite good. Table 1 lists the mean angles for a specific hybridization state as well as the percentage of atoms for that state in a-C using the different models of interaction. A comparison of the concentration of various coordination atoms in a-C networks with those from [7] is also included. 
Table 1. Mean angles of atoms for a specific hybridization state $\left(s p, s p^{2}\right.$ or $\left.s p^{3}\right)$ and the percentage of atoms for that state in a-C using the different interatomic models.

\begin{tabular}{ccccc}
\hline & \multicolumn{3}{c}{ This work } & Stuart et al. [7] \\
\hline & & Mean angle $(\AA)$ & Coordination $(\%)$ & Coordination (\%) \\
\hline \multirow{2}{*}{ AIREBO } & $s p$ & 109.77 & 1.97 & 0.44 \\
& $s p^{2}$ & 109.09 & 88.63 & 89.35 \\
& $s p^{3}$ & 109.46 & 9.40 & 10.20 \\
\hline \multirow{2}{*}{ REBO } & $s p$ & 141.92 & 5.50 & \\
& $s p^{2}$ & 119.03 & 85.90 & \\
& $s p^{3}$ & 109.09 & 8.49 & \\
\hline \multirow{2}{*}{ 2NN-MEAM } & $s p$ & 119.98 & 7.43 & \\
& $s p^{2}$ & 118.17 & 86.63 & \\
\hline
\end{tabular}

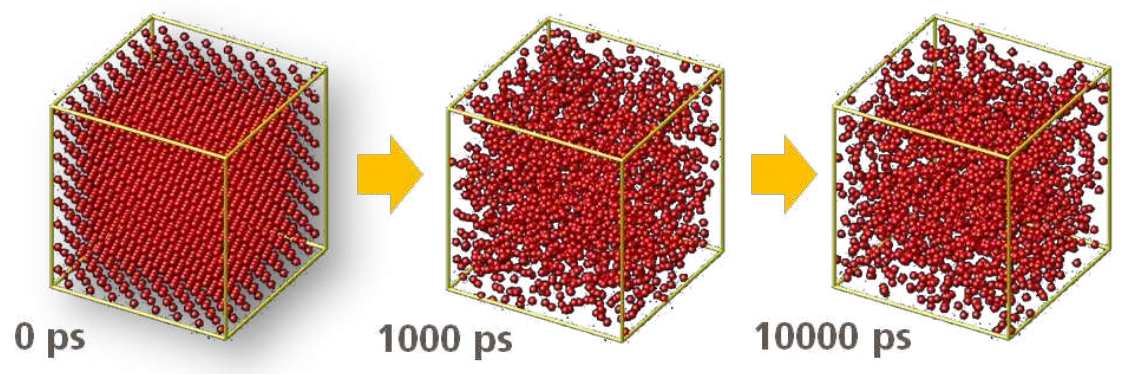

Figure 8. Evolution of the carbon structure in the generation process, from a regular to an amorphous system.

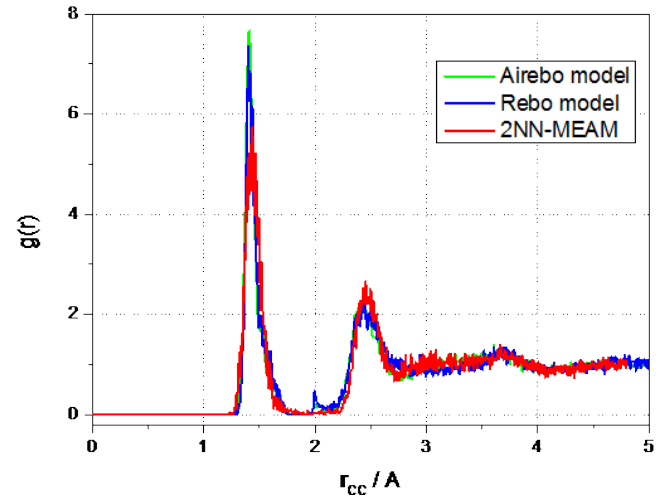

(a)

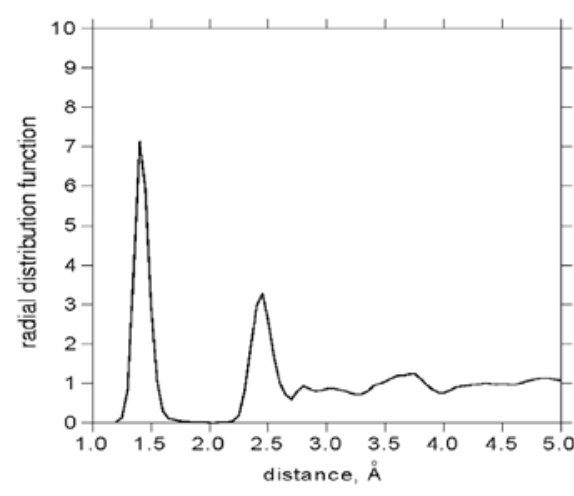

(b)

Figure 9. Calculated C-C RDFs: a) a-C at 2.6 g cc-3 using different potential of interactions, b) previous simulated radial distribution function of a-C (from [30]). 
We decided to continue using the 2NN-MEAM potential due its suitability for covalently bonded materials. Thus, in order to validate the 2NN-MEAM potential for carbon structures, we computed the elastic constants for a diamond structure. Table 2 shows the elastic constants of diamond calculated using the 2NN-MEAM potential, in comparison with other calculations and experiments. The elastic constants are well reproduced by this potential, being $\mathrm{C}_{11}=447 \mathrm{GPa}$, $\mathrm{C}_{12}=72 \mathrm{GPa}$, and $\mathrm{C}_{44}=184 \mathrm{GPa}$. These results are in a good agreement with those published for a pure nanocomposite carbon (na-C) with similar density [8] ( $\mathrm{C}_{11} \sim 500 \mathrm{GPa}, \mathrm{C}_{12} \sim 100 \mathrm{GPa}$, and $\mathrm{C}_{44} \sim 200 \mathrm{GPa}$ ). The stress-strain curve for an a-C sample suggests a ductile behavior as shown in Figure 10. At room temperature, the critical stress level is reached at $20 \mathrm{GPa}$.

Table 2. Elastic constants (in GPa) for diamond

\begin{tabular}{lccccc}
\hline & $\begin{array}{c}\text { This } \\
\text { work }\end{array}$ & REBO [44] & AIREBO [27] & Tersoff [45] & $\begin{array}{c}\text { Experimental data } \\
{[\mathbf{4 4}][\mathbf{4 6}]}\end{array}$ \\
\hline $\mathbf{C}_{\mathbf{1 1}}$ & 1079 & 1070 & 1120 & 1090 & 1079,1080 \\
$\mathbf{C}_{\mathbf{1 2}}$ & 127 & 100 & 130 & 120 & 124,127 \\
$\mathbf{C}_{\mathbf{4 4}}$ & 623 & 680 & 770 & 640 & 578,577 \\
\hline
\end{tabular}

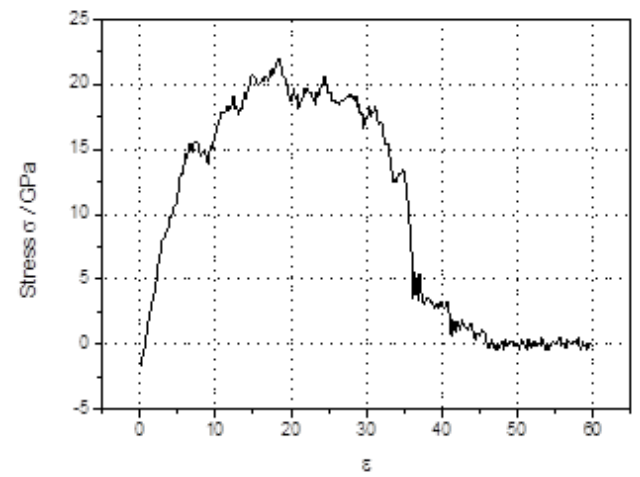

Figure 10. Computed stress-strain curve for uniaxial tension loading on the a-C structure at $300 \mathrm{~K}$. 
In spite of the huge technological interest in amorphous carbon, there are few reports on its coefficient of thermal expansion (CTE). Marques et al. [9] showed that the CTE depends on the concentration of $s p^{2}$ bonded carbon, increasing to the value of graphite as the $s p^{2}$ concentration approaches $100 \%$. In this work, we were able to reproduce the CTE of Wang et al. [47] for an a$\mathrm{C}$ structure where the $90 \%$ of the $\mathrm{C}$ bonds are $s p^{2}$ (quite close to our selected system, $86.63 \%$ ), yielding a value of $6.610^{-6} 1 / \mathrm{K}$ in contrast to $6.810^{-6} 1 / \mathrm{K}$ found in [47].

To create and characterize the TiC phase, the system is defined using an fcc structure with a lattice parameter of $4.42 \AA$. Regarding the mechanical properties, the elastic constants were computed using the 2NN-MEAM potential, reproducing the results by [31], as shown in Table 3 . The stress-strain curve for the TiC structure is shown in Figure 11, showing a maximum at $\sim 30$ GPa.

Regarding, the CTE, a value of $4.64 \cdot 10^{-6} 1 / \mathrm{K}$ was obtained in the $x$ direction. We also computed the coefficient of thermal expansion along the $y$ and $z$ directions of the TiC system resulting $4.20 \cdot 10^{-6} 1 / \mathrm{K}$ and $4.43 \cdot 10^{-6} 1 / \mathrm{K}$, respectively. As expected, the structure has an almost isotropic behavior and the results are within the range provided by Engbergand and Zehms [48]. Moreover, in order to reproduce the conditions of reference [48], a simulation for a wider range of temperature (1200-2900 K) is performed, yielding a value of $8.92 \pm 0.05 \mathrm{~K}^{-1}$, which is also in good agreement with the published one.

Table 3. Elastic constants (in GPa) for TiC.

\begin{tabular}{lcc}
\hline & This work & Kim and Lee [31] \\
\hline $\mathbf{C}_{\mathbf{1 1}}$ & 522 & 522 \\
$\mathbf{C}_{\mathbf{1 2}}$ & 102 & 102 \\
$\mathbf{C}_{\mathbf{4 4}}$ & 129 & 129 \\
\hline
\end{tabular}

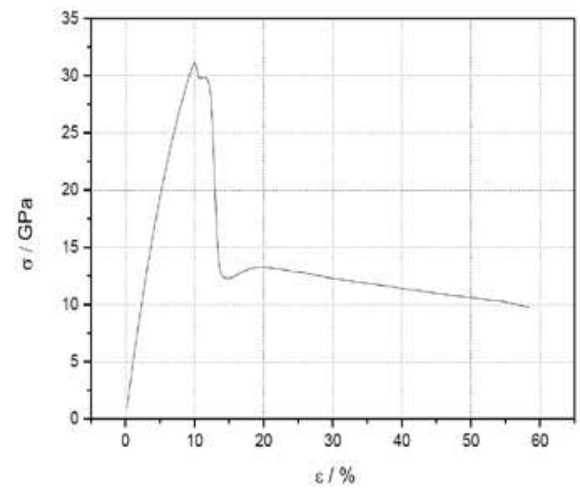

Figure 11. Computed stress-strain curve for uniaxial tension loading on the TiC structure at $300 \mathrm{~K}$. 


\subsection{RVE characterization of nanoabsorbers}

At the nanoscale, or more precisely the submicroscale, the nanoabsorber is presented as a heterogeneous composite material, with an a-C matrix and $\mathrm{TiC}$ embedded particles. Both phases have been characterized at the lowest scale [5]. Thus, this information can be used at the next scale in order to characterize the behavior of the nanoabsorber as a whole.

In order to carry out the thermo-mechanical characterization of the absorber layer, an RVE has to be defined. In this study, we choose a unit cell with a single inclusion and a random distribution of several inclusions meeting the same volume fraction $\left(\mathrm{V}_{\mathrm{f}}=30 \%\right)$. Thus, the former is a cubic specimen of size $6 \mathrm{~nm}$ with a particle with diameter $5 \mathrm{~nm}$; and the latter is a $200 \mathrm{~nm}$ cubic specimen with 9 inclusions of $5 \mathrm{~nm}$ in diameter, as depicted in Figure 12. For both configurations we also model the interface between matrix and inclusion with zero thickness linear elements using thermo-mechanical cohesive damage model [13]. The specimen size has been defined so as to respect the average size of the $\mathrm{TiC}$ particles synthesized in the laboratory and so as to comply with the volume fraction of these particles in the resulting composite material. In any case, as it is shown in reference [42], in first order multiscale procedures, the size of the RVE is not relevant, as long as the proportions between the different materials is fulfilled.

On the other hand, the RVE with 9 inclusions has been defined in order to evaluate the effect of the interaction between the different particles in the material. Moreover, as they have a regular distribution, the results obtained from both models are expected to be very similar. However, in the case of a random distribution of the different particles in the composite, more RVE configurations should be considered so as to ensure the representability of the model, as it is shown in [49].

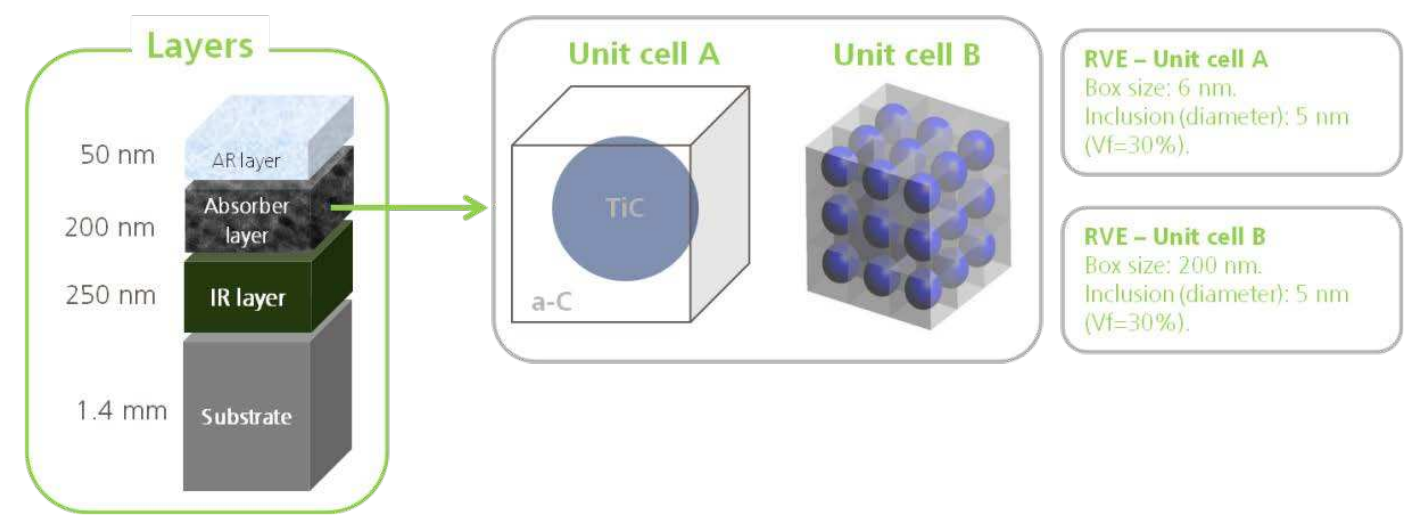

Figure 12. SSC arrangement and nanoabsorber layer RVEs. 
Table 4 shows the thermo-mechanical parameters considered for the characterization of the nano-absorber layer components, namely $\mathrm{TiC}$ and a-C. These properties are applied to the RVE models described previously, obtaining the composite homogenized mechanical properties shown in Table 5. This table shows that the results obtained with the model containing 1 sphere are, as expected, very similar to the results obtained with the 9 spheres model. The values obtained are in agreement with those provided by the rule of mixtures. This formulation provides the properties of the composite averaging the contribution of each component by its volumetric participation $[21,50]$. It combines the components assuming that they have a parallel, serial, or mixed behavior $[51,21]$, and it can also account for material non-linearity $[52,53]$. It must be remarked that, when compared with the homogenized results, the mixing theory usually provides conservative results [43].

Figure 13 presents the Cauchy stress components in the $z$ (transversal) direction for both unit cells subjected to prescribed displacements. It can be seen that the minimum and maximum values are in good agreement in both cases.

Table 4. Thermal and mechanical properties of a-C and TiC, estimated from [8] and [54]. The homogenized absorber properties have been estimated using the rule of mixtures.

\begin{tabular}{ccccccc}
\hline Phase & $\begin{array}{c}\text { Density } \\
\left(\mathbf{k g} / \mathbf{m}^{\mathbf{3}}\right)\end{array}$ & $\begin{array}{c}\text { Conductivity } \\
(\mathbf{W} / \mathbf{m K})\end{array}$ & $\begin{array}{c}\text { Specific. Heat } \\
(\mathbf{J} / \mathbf{k g K})\end{array}$ & $\begin{array}{c}\text { Elastic } \\
\text { Modulus } \\
(\mathbf{G P a})\end{array}$ & $\begin{array}{c}\text { Poisson's } \\
\text { ratio }\end{array}$ & $\begin{array}{c}\mathbf{C T E} \\
(\mathbf{1 0 - 6 / K})\end{array}$ \\
\hline $\mathbf{T i C}$ & 4940 & 5.9 & 710 & 450 & 0.19 & $7.7 \mathrm{e}-6$ \\
$\mathbf{a}-\mathbf{C}$ & 2000 & 1.0 & 500 & 200 & 0.12 & $3.8 \mathrm{e}-6$ \\
$\begin{array}{c}\text { Absorber } \\
(\mathbf{a}-\mathbf{C}+\mathbf{T i C})\end{array}$ & 2900 & 2.5 & 560 & 275 & 0.14 & $5.0 \mathrm{e}-6$ \\
\hline
\end{tabular}

Table 5. Numerical homogenization properties in two simulated unit cells.

\begin{tabular}{ccccc}
\hline Unit Cell & $\begin{array}{c}\text { Elastic modulus } \\
(\mathbf{G P a})\end{array}$ & $\begin{array}{c}\text { Poisson's } \\
\text { ratio }\end{array}$ & $\begin{array}{c}\text { CTE } \\
(\mathbf{1 0 - 6 / K})\end{array}$ & $\begin{array}{c}\text { Conductivity } \\
\text { (W/mK) }\end{array}$ \\
\hline A (1 Sphere) & 256 & 0.140 & 4.96 & 2.298 \\
B (9 Spheres) & 254 & 0.138 & 4.90 & 2.297 \\
Rule of mixtures & 275 & 0.140 & 5.00 & 2.500 \\
\hline
\end{tabular}




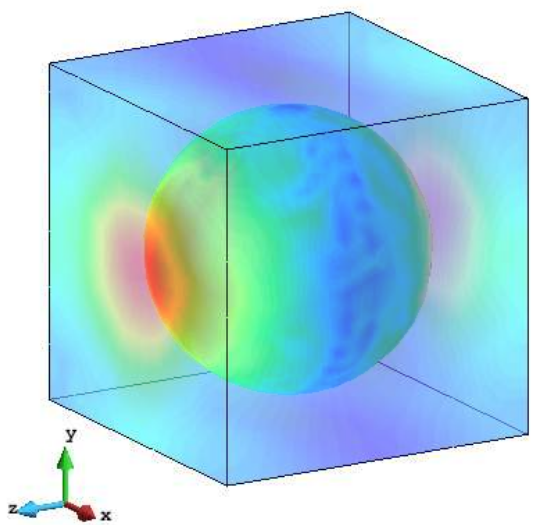

(a)

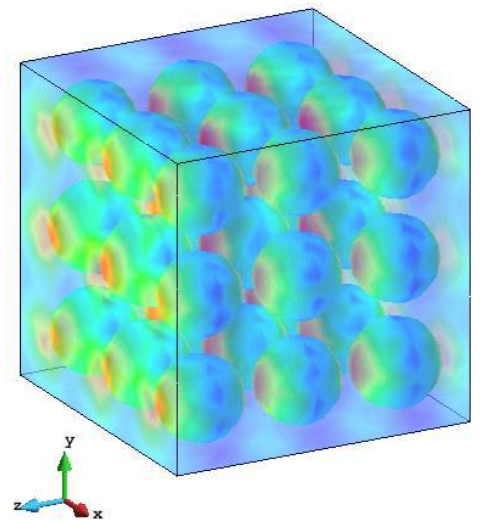

(c)

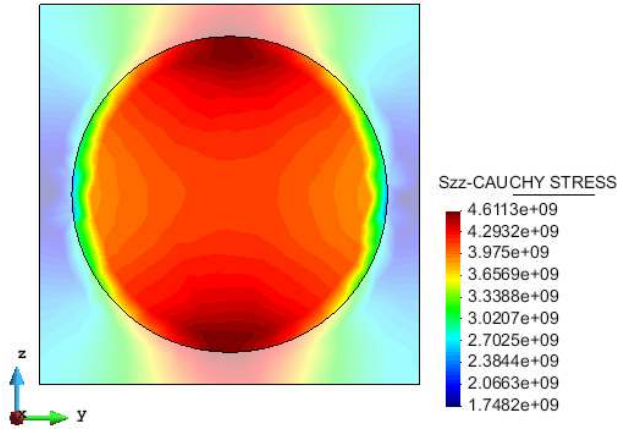

(b)

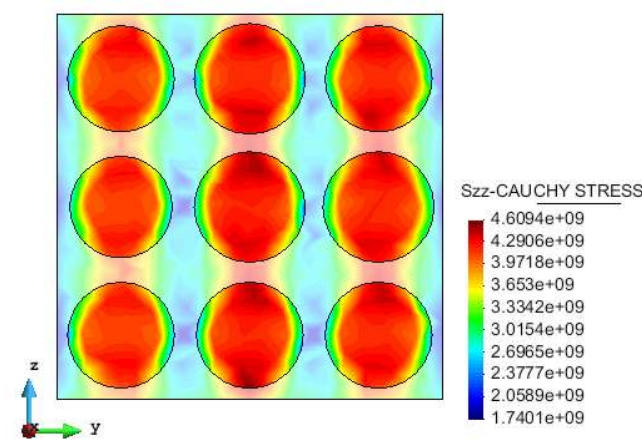

(d)

Figure 13. Stress field at the RVE: a-b) unit cell A, and c-d) unit cell B.

An important issue in this type of nanoabsorber is the potential particle-matrix debonding that may take place with the rise of temperature. The computational methodology developed in this work is able to account for such phenomenon; not only allowing for the debonding itself via cohesive elements, but also including the potential matrix damage (Figure 14). As previously mentioned, we must remark that we did not compute the cohesive laws at the atomistic level. On the other hand, we use the model proposed by Özdemir et. al [13] for illustrative purposes. The material parameters used for the cohesive element are the same of the matrix reduced by $10 \%$. It is also interesting to observe that the predictions of our model could be used at a later stage to characterize a (homogenized) critical stress, beyond which coarse scale damage will begin to develop. This will open the way for a completely ab-initio characterization of the material which is of obvious interest to industry. 
One important effect of particle-matrix debonding is the global loss in the conductivity of the layer as a result of the temperature field redistribution, as observed in Figure 15. This effect can be intuitively understood as newly opened gaps that severely impact the heat conduction across the RVE, due to the appearance of reduced conductivity spots, resulting in an anisotropic reduction of the conductivity in the direction of the opening gaps. The introduction of such effects in a more traditional approach (typically based on closed-form constitutive laws) would be extremely complex since it would require the characterization of a sharp change in the characteristics of the material which is moreover strongly anisotropic in nature.

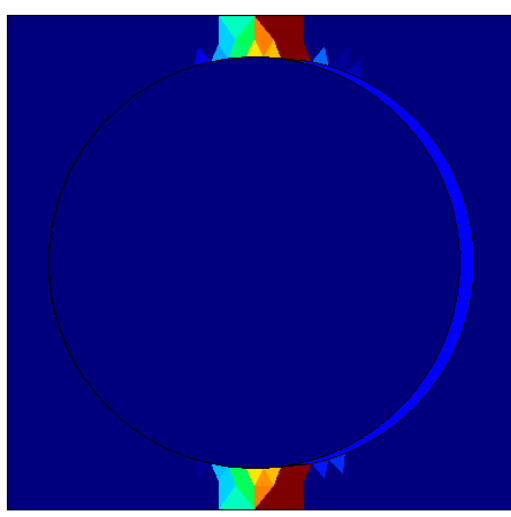

(a)

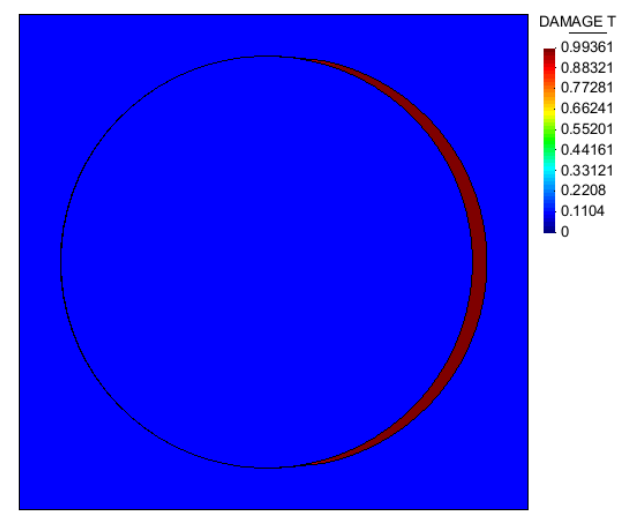

(b)

Figure 14. Particle-matrix debonding in the nanoabsorber: a) matrix damage, and b) cohesive damage).

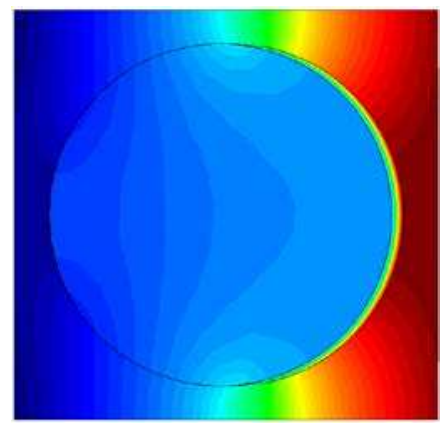

(a)

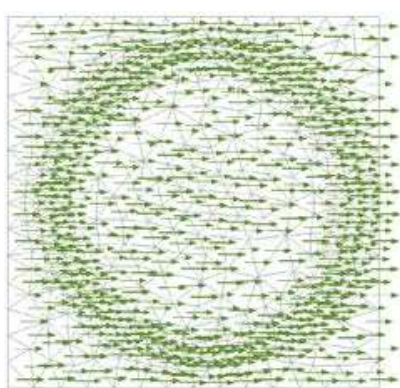

(c)

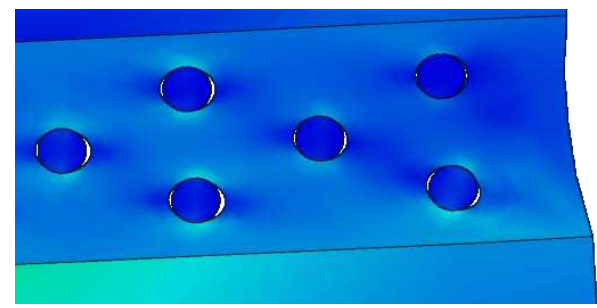

(b)

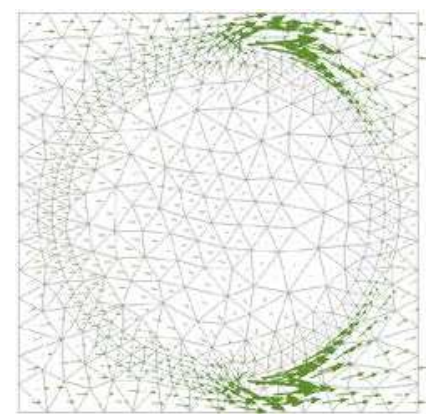

(d)

Figure 15. Particle-matrix debonding issues: a) redistribution of temperature gradient field, and b) global loss of conductivity; and change in the heat flux redistribution: c) before the particle-matrix debonding, and d) after the particle-matrix debonding. 


\subsection{Macroscopic response}

As it is described in Section 5, the macroscopic analysis of the receiver tube is conducted within the linear regime, using the material properties obtained from the RVE simulations. Therefore, the material values obtained above, along with the material information corresponding to the other layers of the tube, it is possible to analyze the macroscale structure (i.e. a piece of the receiver tube) subjected to a given radial thermal gradient as depicted in Figure 16a, that results in the consequent skew deformation shown in Figure $16 \mathrm{~b}$.

Considering the thickness of SSCs negligible with respect to that of the substrate, we can assume the same strain field for the different layers that compose the tube. In that way, using the material properties in Table 6 and simulating the layers as $2 \mathrm{D}$ membranes covering the $3 \mathrm{D}$ substrate, it is possible to determine the stress distribution in any single layer (Figure 17) and foresee potential critical zones.

Table 6. Thermal and mechanical properties used for the different layers.

\begin{tabular}{ccccccc}
\hline Phase & $\begin{array}{c}\text { Density } \\
\left(\mathbf{k g} / \mathbf{m}^{\mathbf{3}}\right)\end{array}$ & $\begin{array}{c}\text { Conductivity } \\
(\mathbf{W} / \mathbf{m K})\end{array}$ & $\begin{array}{c}\text { Specific heat } \\
(\mathbf{J} / \mathbf{k g K})\end{array}$ & $\begin{array}{c}\text { Elastic } \\
\text { modulus } \\
(\mathbf{G P a})\end{array}$ & Poisson's ratio & $\begin{array}{c}\mathbf{C T E} \\
(\mathbf{1 0}-\mathbf{6} / \mathbf{K})\end{array}$ \\
\hline $\begin{array}{c}\mathbf{A R}\left(\mathbf{A l}_{2} \mathbf{O}_{3}\right) \\
\mathbf{A b s o r b e r} \\
(\mathbf{R V E})\end{array}$ & 3900 & 15 & 880 & 340 & 0.22 & 8.1 \\
$\begin{array}{c}\text { IR (TiN) } \\
\text { Substrate } \\
\text { (Inconel) }\end{array}$ & 5220 & 2.5 & 560 & 275 & 0.14 & 4.9 \\
\hline
\end{tabular}

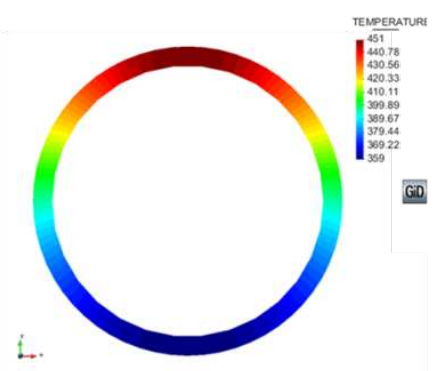

(a)

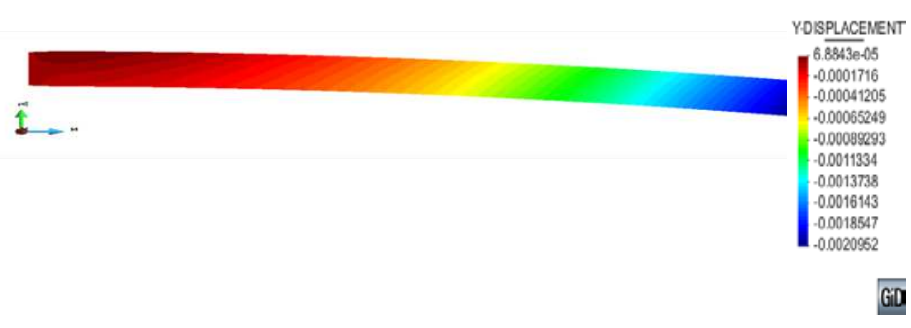

(b)

Figure 16. Absorber tube temperature-induced deformation: a) temperature gradient, and b) vertical displacement field. 

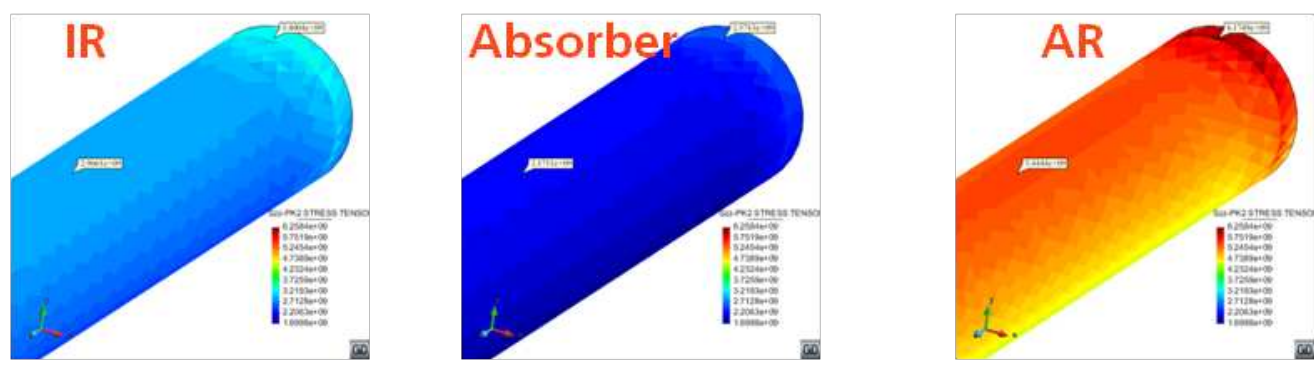

Figure 17. Stress field (Cauchy stress tensor, $z$ component) at different layers.

\subsection{Material top-down analysis}

Although the structural simulation of the receiver tube does not reach the non-linear regime, it does provide the critical zones in which the maximum stress and/or temperature values are found. The results of the Gauss points in these regions can be downscaled, applying the corresponding strain and thermal fields to the RVE in order to determine the microscopic stress distribution and predict potential failure (e.g., cracking, debonding). This feature is already implemented in an inhouse tool for designing micro-materials (GiD-VMD), developed by CIMNE in collaboration with Abengoa Research [25, 55].

For our current analysis, the material response provided by the RVE at some Gauss points is shown in Figure 18. As can be seen in this figure, the RVE has a linear response for all the Gauss points and, therefore, the non-linear response that can be predicted by the RVE, as shown in section 6.2, does not take place.

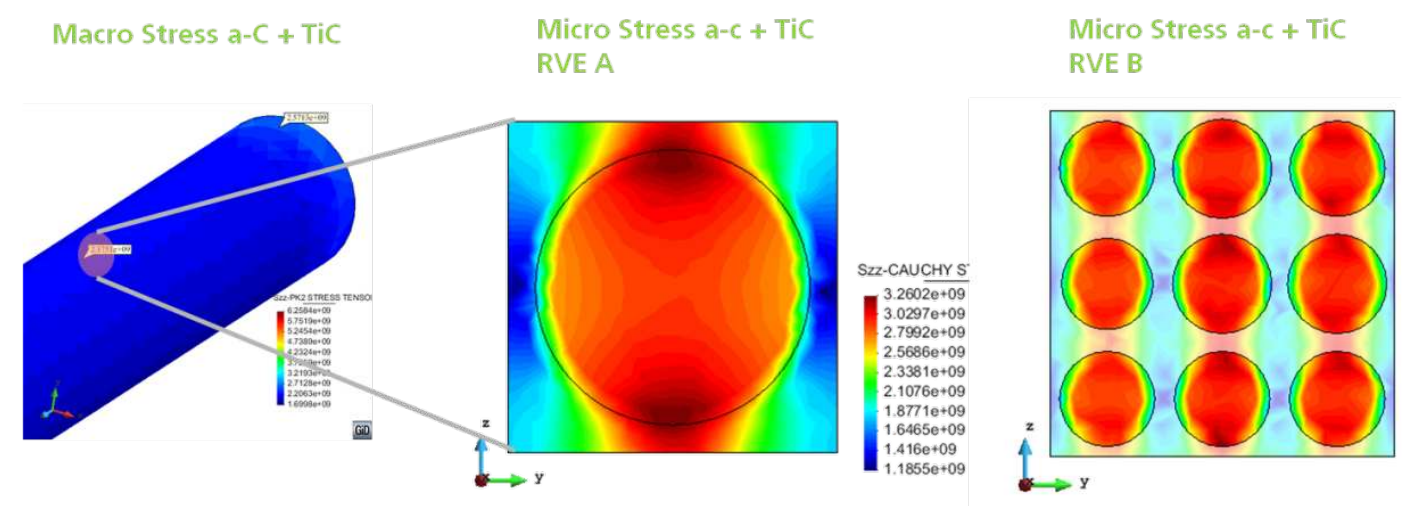

Figure 18. Downscaling from the absorber tube to the RVE level. 


\section{Conclusions}

In the present work, the authors present a multiscale and multiphysical methodology for the analysis of receiver tubes with multi-layered coatings, especially recommended in concentrating solar power plants. On one hand, three different scales are covered, going from the atomistic (with the use of MD) to the structural analysis, through an intermediate microscopic analysis of absorber layers, via FEM simulations. On the other hand, the methodology includes the possibility to account for thermo-mechanical simulations, of main importance in this type of plants. This multiscale approach is valid for the whole elastic regime; however, in the presence of damage localization, it must be carefully revisited. The scales are connected in a hierarchical fashion, being able to cross from the lowest level towards the highest, and the other way around.

Regarding the atomistic simulations, an MD methodology has been successfully applied in order to analyze the material properties of single phases present in nanoabsorbers. In this sense, original results for amorphous carbon, especially for the elastic constants and the CTE, have been derived.

At the microscale, an RVE-based thermo-mechanical homogenization scheme has been presented. It takes into account both the mechanical and the heat transfer problem, being able to provide homogenized mechanical and thermal properties of the nanocomposite. Moreover, it has been validated with experimental results and it has been finally applied to a relevant problem in CSP plants.

Finally, a full multiscale problem, covering the aforementioned scales, has been presented, allowing the identification of critical zones in specific layers of solar selective coatings. The material properties are obtained from fundamental levels, and sequentially upscaled into higherlevel analyses. The definition of the interaction potentials, which is essential in the success of this methodology, is not straightforward as it requires the expertise of the computational material scientists and is time-consuming. However, provided the number of material configurations will exponentially increase in the coming years, the interatomic potential database will progressively be more complete and, thus, actual in silico design of materials will become possible. Of course, as the scale resolution increases, the computational demand also increases. For this reason, efforts should be directed towards numerical methods that will enable complex non-linear multiphysical analysis of these materials.

\section{Acknowledgements}

The authors would like to acknowledge Abengoa Research for partially funding this work within the framework of the "Virtual Materials Design" project. This work has been also financially supported by CIMNE together with the European Community under grant: FP7-PEOPLE-2013- 
IRSES 612607 TCAiNMaND "Tri Continental Alliance in Numerical Methods applied to Natural Disasters", by European Research Council through of Advanced Grant: ERC-2012-AdG 320815 COMP-DES-MAT “Advanced tools for computational design of engineering materials", and by the Dirección General de Investigación Científica y Técnica through the research project: MAT2014-60647-R OMMC “Optimización multi-escala y multi-objetivo de estructuras de laminados compuestos". All this support is gratefully acknowledged.

\section{References}

[1] H. Price, E. Lüpfert, D. Kearney, E. Zarza, E. Cohen, R. Gee and R. Mahoney, "Advances in parabolic trough solar power technology," Journal of Solar Energy Engineering, vol. 124, no. 2, pp. 109-125, 2002.

[2] M. Montes, A. Abánedes, J. Martínez-Val and M. Valdés, "Solar multiple optimization for a solar-only thermal power plant, using oil as heat transfer fluid in the parabolic trough collectors," Solar Energy, vol. 83, no. 12, pp. 2165-2176, 2009.

[3] C. Atkinson, C. Sansom and H. S. C. Alomnd, "Coatings for concentrating solar systems A review," Renewable and Sustainable Energy Reviews, vol. 45, pp. 113-122, 2015.

[4] N. Selvakumar and H. Barshilia, "Review of physical vapor deposited (PVD) spectrally selective coatings for mid- and high-temperature solar thermal applications," Solar Energy Materials and Solar Cells, vol. 98, pp. 1-23, 2012.

[5] I. Heras, M. Krause, G. Abrasonis, A. Pardo, J. Endrino, E. Guillén and R. EscobarGalindo, "Advanced characterization and optical simulation for the design of solar selective coatings based on carbon: transition metal carbides," Solar Energy Materials and Solar Cells, vol. 157, pp. 580-590, 2016.

[6] Abengoa Research, "Abengoa Research Strategic Research Agenda," Abengoa (Internal report), Seville, Spain, 2012.

[7] S. Stuart, M. Knippenberg, O. Kum and P. Krstic, "Simulation of amorphous carbon with a bond-order potential," Physica Scripta, vol. T124, pp. 58-64, 2006.

[8] I. Remediakis, M. Fyta, C. Mathioudakis, G. Kopidakis and P. Kelires, "Structure elastic properties and strength of amorphous and nanocomposite carbon," Diamond and Related Materials, vol. 16, no. 10, pp. 1835-1840, 2007.

[9] F. Marques, R. Lacerda, A. Champi, V. Stolojan, D. Cox and S. Silva, "Thermal expansion coefficient of hydrogenated amorphous carbon," Applied Physics Letters, vol. 83, no. 15, pp. 3099-3101, 2003.

[10] E. J. Barbero, Introduction to composite materials design, 2nd Edition, Taylor \& Francis, 2011.

[11] S. Oller, J. Canet and F. Zalamea, "Composite material behavior using a homogenization double scale method," Journal of Engineering Mechanics, vol. 131, no. 1, pp. 65-79, 2005. 
[12] I. Özdemir, W. Brekelmans and M. Geers, "FE2 computational homogenization for the thermo-mechanical analysis of heterogeneous solids," Computer Methods in Applied Mechanics and Engineering, vol. 198, no. 3-4, pp. 602-613, 2008.

[13] I. Özdemir, W. Brekelmans and M. Geers, "A thermo-mechanical cohesive zone model," Computational Mechanics, vol. 46, no. 5, pp. 735-745, 2010.

[14] P. Kanouté, D. Boso, J. Chaboche and B. Schrefler, "Multiscale methods for composites: A review," Archives of Computational Methods in Engineering, vol. 16, pp. 31-75, 2009.

[15] V. Nguyen, M. Stroeven and L. Sluys, "Multiscale failure modelling of concrete: micromechanical modelling, discontinuous homogenization and parallel computations," Computer Methods in Applied Mechanics and Engineering, Vols. 201-204, pp. 139-156, 2012.

[16] C. Dandekar and Y. Shin, "Molecular dynamics based cohesive zone law for describing Al-SiC interace mechanics," Composites Part A: Applied Science and Manufacturing Volume, vol. 42, no. 4, pp. 355-363, 2011.

[17] X. Dong and Y. Shin, "Coupled thermomechanical multiscale modeling of alumina ceramics to predict thermally induced fractures under laser heating," Journal of the American Ceramics Society, vol. 98, no. 3, pp. 920-928, 2015.

[18] A. Karamnejad, V. Nguyen and L. Sluys, "A multi-scale rate dependent crack model for quasi-brittle heterogeneous materials," Engineering Fracture Mechanics, vol. 104, pp. 96113, 2013.

[19] F. Montero-Chacón, E. García-Pérez, J. Sanz-Herrera and M. Doblaré, "Virtual Materials Design platform in sustainable solutions," in 1st International Workshop on Software Solutions for Integrated Computational Materials Engineering, Rolduc, The Netherlands, 2014.

[20] Committee on Integrated Computational Materials Engineering, National Materials Advisory Board, Division on Engineering and Physical Sciences, National Research Council, "Integrated Computational Materials Engineering: A Transformational Discipline for Improved Competitiveness and National Security," National Academies Press, 2008.

[21] S. Oller, Numerical simulation of mechanical behaviour of composite materials, Barcelona: CIMNE - Springer, 2014.

[22] S. Ghosh, K. Lee and S. Moorthy, "Two scale analysis of heterogeneous elastic-plastic materials with asymptotic homogenization and Voronoi cell finite element model," Computer Methods in Applied Mechanics and Engineering, no. 132, p. 63-116, 1996.

[23] M. Horstemeyer, Integrated computational materials engineering (ICME) for metals: using multiscale modeling to invigorate engineering design with science, Hoboken, New Jersey: John Wiley \& Sons, Inc., 2012.

[24] S. Plimpton, "Fast parallel algorithms for short-range molecular dynamics," Journal of Computational Physics, vol. 117, pp. 1-19, 1995.

[25] CIMNE, "GiD-LAMMPS: GiD problem type for LAMMPS molecular dynamics code," CIMNE, Barcelona, Spain, 2015.

[26] D. Brenner, "Empirical potential for hydrocarbons for use in simulating the chemical vapor deposition of diamond films," Physical Review B, vol. 42, no. 15, pp. 9458-9471, 1990 . 
[27] S. Stuart, A. Tutein and J. Harrison, "A reactive potential for hydrocarbons with intermolecular interactions," The Journal of Chemical Physics, vol. 112, no. 14, pp. 64726486, 2000.

[28] B.-J. Lee and M. Baskes, "Second nearest-neighbor modified embedded-atom-method potential," Physical Review B, vol. 62, no. 13, pp. 8564-8567, 2000.

[29] B.-J. Lee, M. Baskes, H. Kim and Y. Cho, "Second nearest-neighbor modified embedded atom method potentials for bcc transition metals," Physical Review B, vol. 64, no. 18, p. 184102, 2001.

[30] B.-J. Lee and J. Lee, "A modified embedded atom method interatomic potential for carbon," Calphad-Computer Coupling of Phase Diagrams and Termochemistry, vol. 29, no. 1, pp. 7-16, 2005.

[31] Y.-M. Kim and B.-J. Lee, "Modified embedded-atom method interatomic potentials for the Ti-C and Ti-N binary systems," Acta Materialia, vol. 56, no. 14, pp. 3481-3489, 2008.

[32] F. Müller-Plathe, "Reversing the perturbation in nonequilibrium molecular dynamics: An easy way to calculate the shear viscosity of fluids," Physical Review E, vol. 59, no. 5, pp. 4894-4898, 1999.

[33] J. M. Ortolano, J. Hernández and J. Oliver, A comparative study on homogenization strategies for multi-scale analysis of materials, Barcelona, Spain: International Center for Numerical Methods in Engineering, February 2013.

[34] F. Feyel and J.-L. Chaboche, "FE2 multiscale approach for modelling the elastoviscoplastic behaviour of long fibre $\mathrm{SiC} / \mathrm{Ti}$ composite materials," Computer Methods in Applied Mechanics and Engineering, vol. 183, no. 3-4, pp. 309-330, 2000.

[35] S. F. I. G. V. M. a. D. J. T. Kanit, "Determination of the size of the representative volume element for random composites: statistical and numerical approach.," Int. J. Solids Struct., pp. 40, 13-14:3647-3679, 2003.

[36] M. O.-S. a. I. J. M. Jiang, "Scale-dependent bounds on effective elastoplastic response of random composites.," J. Mech. Phys. Sol., pp. 49:655-673, 2001.

[37] M. O.-S. a. I. J. M. Jiang, "Apparent elastic and elastoplastic behavior of periodic composites.," Int. J. Solids Struct., pp. 39:199-212, 2002.

[38] M. Ostoja-Starzewski, "Material spatial randomness: From statistical to representative volume element.," Prob. Eng. Mech., pp. 21:112-132, 2006.

[39] F. Otero, X. Martinez, S. Oller and O. Salomón, "An efficient multi-scale method for nonlinear analysis of composite structures," Composite Structures, vol. 131, no. 1, pp. 707$719,2015$.

[40] M. Petracca, L. Pelà, R. Rossi, S. Oller, G. Camata and E. Spacone, "Regularization of first order computational homogenization for multiscale analysis of masonry structures," Computational Mechanics, vol. 57, no. 2, pp. 257-276, 2016.

[41] A. Melro, P. Camanho, F. Andrade Pires and S. Pinho, "Numerical simulation of the nonlinear deformation of 5-harness satin weaves," Computational Materials Science, vol. 61, p. 116-126, 2012.

[42] F. Otero, S. Oller and X. Martínez, "Multiscale Computational Homogenization: Review and Proposal of a New Enhanced-First-Order Method," Archives of Computational Methods in Engineering, 2016. 
[43] F. Otero, S. Oller, X. Martinez and O. Salomón, "Numerical homogenization for composite materials analysis. Comparison with other micro mechanical formulations," Composite Structures, vol. 122, pp. 405-416, 2015.

[44] D. Brenner, O. Shenderova, J. Harrison, S. Stuart, B. Ni and S. Sinnott, "A secondgeneration reactive empirical bond order (REBO) potential energy expression for hydrocarbons," Journal of Physics-Condensed Matter, vol. 14, no. 4, pp. 783-802, 2002.

[45] J. Tersoff, "Empirical interatomic potential for carbon with applications to amorphouscarbon," Physical Review Letters, vol. 61, no. 25, pp. 2879-2882, 1988.

[46] R. Vogelsang, A. Ramdas, S. Rodriguez, M. Grimsditch and T. Anthony, "Brillouin and Raman scattering in natural and isotopically controlled diamond," Physical Review B, vol. 54, no. 6, pp. 3989-3999, 1996.

[47] J. Wang, Y. Sugimura, A. Evans and W. Tredway, "The mechanical performance of DLC films on steel substrates," Thin Solid Films, vol. 325, no. 1-2, pp. 163-174, 1998.

[48] C. Engberg and E. Zehms, "Thermal expansion of Al2O3, BeO, MgO, B4C, SiC and TiC above $1000{ }^{\circ}$ C," Journal of the American Ceramic Society, vol. 42, no. 6, pp. 300-305, 1959.

[49] I. Gitman, H. Askes and L. Sluys, "Representative volume: Existence and size determination," Engineering Fracture Mechanics, vol. 74, no. 16, pp. 2518-2534, 2007.

[50] X. Martinez and S. Oller, "Numerical simulation of matrix reinforced composite materials subjected to compression loads," Arhives of computational methods in engineering, vol. 16, no. 4, pp. 357-397, 2009.

[51] F. Rastellini, S. Oller, O. Salomon and E. Oñate, "Composite materials non-linear modelling for long fibre-reinforced laminates: Continuum basis, computational aspects and validations," Computers \& Structures, vol. 86, no. 9, pp. 879-896, 2008.

[52] X. Martinez, S. Oller and E. Barbero, "Study of delamination in composites by using the serial/parallel mixing theroy and a damage formulation," in Mechanical response of composites, Springer, 2008, pp. 119-140.

[53] X. Martinez, S. Oller, F. Rastellini and A. H. Barbat, "A numerical procedure simulating RC structures reinforced with FRP using the serial/parallel mixing theory," Computers \& Structures, vol. 86, no. 15-16, pp. 1604-1618, 2008.

[54] H. Pierson, Handbook of refractory carbides and nitrides: Properties, characteristics, processing and applications., Westwood, NJ: Noyes Publications, 1996.

[55] Abengoa Research, "Benchmarks in renewable energies conversion for the Virtual Materials Design platform," Abengoa (Internal report), Seville, Spain, 2014. 